\title{
Emphysematous pyelonephritis, case report and review of the literature
}

\begin{abstract}
Background: Urinary tract infections in immunosuppressed patients, especially Diabetic ones with poor control, can progress to a severe necrotizing infection called emphysematous pyelonephritis (EP), a diagnosis that must be highly suspected in these cases for the presentation as a simple urinary tract infection with poor response to medical management. Delayed diagnosis increases the already high morbidity and mortality.
\end{abstract}

Case: We present the case of a 47-year-old female admitted to emergency department for a 4day disease characterized by fever, chills, adynamia, asthenia and right flank pain of moderate intensity. Past day she refers hematemesis in an occasion. She denied pathologic background. At physical examination with inflammatory response signs, right flank pain, positive Giordano sign and positive superior ureteral points, without signs of peritoneal irritation. Laboratories report with blood cells $13000 / \mathrm{mm} 3$, Hemoglobin $13.7 \mathrm{~g} / \mathrm{dL}$, Platelets 82000, Glucose 595mg/dL, Cr 2mg/dL. Gas Analysis report metabolic acidosis. Urinalysis report leucocytes $>75 \mu \mathrm{L}$, uncountable red blood cells, bacteries +++ , nitrites +++ Glucose +++ , proteins + , ketones ++ . Pyelonephritis and Diabetes mellitus 2 of recent diagnosis was established. After twodays of medical treatment she presented septic shock with acute renal failure AKIN II, hematological and hepatic disfunction. Mechanic ventilatory support and use of norepinephrine were adhering to therapy. This case was present in a rural hospital and the unique imaging auxiliary available was an abdomen X-ray that shows an air bubble in the right perirenal space. The diagnosis of emphysematous pyelonephritis was settled down and urgent right nephrectomy performed after platelet transfusion. The surgery was successful and without complications finding a right kidney of 9x6 4 centimeters with multiple parenchymal abscesses. After 11days patient was discharged uneventfully.

Conclusion: The diagnosis of EP must be highly suspected in Diabetes mellitus 2patients with severe urinary tract infection and poor response to initial medical management. Medical management including parenteral antibiotics plus percutaneous catheter drainage or nephrectomy must be performed immediately according to the risk factors by the high morbidity and mortality associated with this disease.

Keywords: sepsis, emphysematous pyelonephritis, nephrectomy, percutaneous, urinary tract, leucocytes, diabetic, ep, ketoacidosis, parenchymal, morbidity, mortality, nephrectomized, acute renal failure
Volume 3 Issue 3 - 2015

\author{
Luis Angel Medina Andrade,' Stephanie \\ Serrano Collazos,' Maria de los Angeles \\ Martinez Ferretiz,' Brenda Ruiz,' Maria \\ Fernanda Chein Vazquez,' Sheila Anahi \\ Urbina Hernandez, ${ }^{2}$ Lizette Blanco Aguilar, ${ }^{2}$ \\ Arianna Alvaró Hernández ${ }^{2}$ \\ 'Department of General Surgery, Universidad de Quintana Roo, \\ Mexico \\ ${ }^{2}$ Hospital Rural Prospera, Instituto Mexicano del Seguro Social, \\ Mexico
}

Correspondence: Luis Angel Medina Andrade, Department of General Surgery, Universidad de Quintana Roo, Instituto Mexicano del Seguro Social, Hospital General Regional No. 17, Servicio de Cirugía General,Av. Politécnico Manzana I Lote I Región 509 C.P. 55750, Cancún, Quintana Roo, Mexico, Tel (+52) (55) 9981963197, (+55) 998। I14201,

Email buismedina_5@hotmail.com

Received: November 9, 2015 | Published: November 30, 2015
Abbreviations: EP, emphysematous pyelonephritis; DKA, diabetic ketoacidosis; CT, computed tomography

\section{Introduction}

Emphysematous pyelonephritis (EP) is defined as a severe necrotizing infection with gas formation in the renal parenchyma or perirenal tissues. It is more common in females than males $(3: 1)$, left side involvement $(60 \%)$ and is present as a complication of urinary infections in Diabetic patients in $90 \%$ of cases. ${ }^{1}$ The diagnosis of this entity must be highly suspected in poor controlled Diabetic patients with urinary tract infection and poor response to medical treatment. In this moment imaging studies like TAC can be developed to discard this life threatening complication. ${ }^{2}$

\section{Case presentation}

We present the case of a 47-year-old female admitted to emergency department for a 4day disease characterized by fever, chills, adynamia, asthenia and right flank pain of moderate intensity. Past day she refers hematemesis in an occasion. She denied pathologic background. At physical examination she presents with $39^{\circ} \mathrm{C}$, arterial tension $90 / 60 \mathrm{mmHg}$, 95 hearths per minute, 23 breaths per minute, saturation $91 \%$, right flank pain, positive Giordano sign and positive superior ureteral points, without signs of peritoneal irritation. Laboratories report withe blood cells $13000 / \mathrm{mm}^{3}$, neutrophils $92 \%$, Hemoglobin $13.7 \mathrm{~g} / \mathrm{dL}$, Platelets 82000 , Glucose $595 \mathrm{mg} / \mathrm{dL}$, Cr $2 \mathrm{mg} / \mathrm{dL}$. Gas Analysis report $\mathrm{pH} 7.28 \mathrm{HCO} 3-7 \mathrm{mmol} / \mathrm{L}$ BEecf $-19.7 \mathrm{mmol} / \mathrm{L}, \mathrm{SO}_{2}$ $88 \%$. Urinalysis report leucocytes $>75 \mu \mathrm{L}$, uncountable red blood cells, bacteries +++ , nitrites +++ Glucose +++ , proteins + , ketones ++ . Pyelonephritis and Diabetes mellitus ${ }^{2}$ of recent diagnosis was established. After twodays of medical treatment she presented septic shock with acute renal failure AKIN II, hematological and hepatic disfunction. Mechanic ventilatory support and use of norepinephrine were adhere to therapy. Laboratories report with blood cells $8400 /$ $\mathrm{mm}^{3}$, neutrophils $82 \%$, Hemoglobin $9.5 \mathrm{~g} / \mathrm{dL}$, Platelets 57000 , Glucose $595 \mathrm{mg} / \mathrm{dL}, \mathrm{Cr} 2 \mathrm{mg} / \mathrm{dL}$, total bilirubin $6.3 \mathrm{mg} / \mathrm{dL}$, lactic dehydrogenase $572 \mathrm{U} / \mathrm{l}$, alanine aminotransferase $352 \mathrm{U} / \mathrm{l}$. This case was present in a rural hospital and the unique imaging auxiliary available was an 
abdomen X-ray that shows an air bubble in the right perirenal space (Figure 1). Emphysematous pyelonephritis was diagnosed and urgent right nephrectomy performed after platelet transfusion. The surgery was successful and without complications finding a right kidney of $9 \times 6 \times 4$ centimeters with multiple parenchymal abscesses (Figure 2). After 6days extubation was achieved by internal medicine service and after 5days the patient was discharged uneventfully.

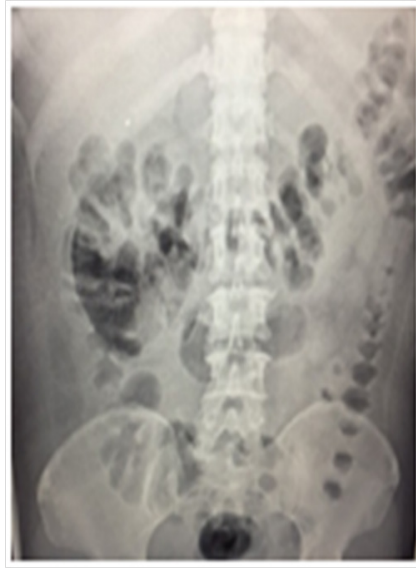

Figure I A) Abdomen X-ray showing a bubble in the perirenal right space.

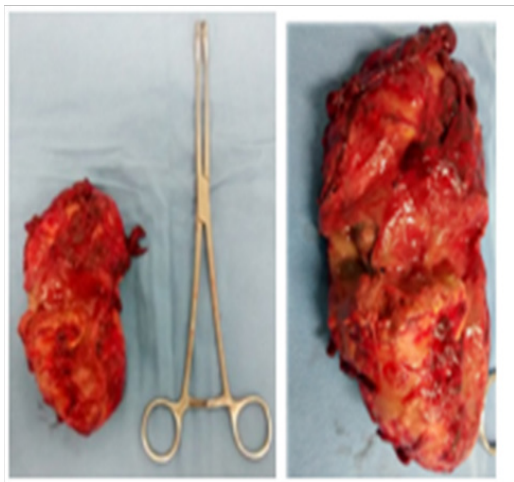

Figure 2 A) Right Kidney with multiple parenchymal abscesses rupture to the surface.

\section{Discussion}

Emphysematous pyelonephritis (EP) is more common among females than males $(3: 1)$ with left side involvement in $60 \%$ of cases. ${ }^{3}$ It is a rare life-threatening infection producing abscess with intrarenal gas, vasculitis, intravascular hemolysis, thrombosis with infarction, papillary necrosis and glomerular sclerosis. It will presents with symptoms like fever, pain and pyuria in about $70 \%$ of cases. When septic shock was present it was associated with a mortality of $54.4 \%$ in some studies and it should be managed promptly with a multidisciplinary approach. ${ }^{3}$ Mortality rate is about $18 \%$ and must be suspected in Diabetic patients with acute pyelonephritis poor response to medical treatment, otherwise the clinical course may be life-threatening if not recognized..$^{2}$ The presentation of this entity in Diabetic patients is secondary to the hydrogen and carbon dioxide release through sugar fermentation by the higher glucose concentrations in tissues, which favors the growth of microorganisms ${ }^{4}$ including E. coli in $51 \%$, followed by Klebsiella $18 \%$ and Pseudomonas $13 \% 5$. Thrombocytopenia, acute renal failure, shock and conservative treatment have been identified as significant risk factors for mortality. ${ }^{5,6}$ Diabetic ketoacidosis (DKA) is an uncommon presentation; only few cases of DKA and EP have been reported in the literature. The finding of gas within renal structures is pathognomonic of EP. ${ }^{7}$ The clinical presentation and laboratory findings must represent an acute pyelonephritis and for this reason the diagnosis requires imaging studies. In X-ray images gas bubbles are observed on renal parenchyma or perirenal tissues, psoas muscle is effaced. Ultrasound must be inadequate in settle down EP diagnosis. ${ }^{4}$ Computed Tomography (CT) scanning is the gold standard to diagnosis and classification, this last based on the extension of gas and location around the kidney ${ }^{7}$ (Table 1).

Table I Huang and Tseng classification of EP CT scan findings (2000)

\begin{tabular}{ll}
\hline Class I & Gas confined to the collecting system \\
Class 2 & Gas confined to the renal parenchyma alon \\
Class 3A & Perinephric extension of gas or abscess \\
Class 3B & Extension of gas beyond the Gerota fascia \\
Class 4 & Bilateral EP or EP in a solitary kidney
\end{tabular}

EP therapy is controversial. Evann off reported mortality rates of $80 \%$ in patients treated with medical management, $60 \%$ in patients who undergone percutaneous drainage and $20 \%$ in nephrectomized patients. ${ }^{4}$ Shoiker et al. reported a series of 15 patients that were nephrectomized after stabilization with an overall mortality of $20 \% 4$. Several investigators have proposed nephrectomy as them in treatment of patients with this life threatening infection. Percutaneous drainage, relief of urinary tract obstruction, antimicrobial treatment and nephrectomy are possible therapeutic strategies according to the clinical course, whether benign or fulminant. Matthew E Falangas et al., ${ }^{6}$ in agreement with Ahlering and Shokeir suggest that conservative treatment is a risk factor that increases mortality in patients with EP. ${ }^{6}$ Percutaneous drainage and medical management were associated with significantly higher survival rates than nephrectomy in some series. ${ }^{3}$ Tahsin Turunc ${ }^{8}$ and Sang Hyun Park et al., ${ }^{9}$ suggest percutaneous catheter drainage and interval nephrectomy as the gold standard treatment, ${ }^{8,9}$ but even with the great advances in minimally invasive surgery and renal preservation therapies, open nephrectomy has a valuable role in the EP management, especially in places without access to this treatment options. ${ }^{1,10}$

\section{Conclusion}

The diagnosis of EP must be highly suspected in Diabetes mellitus 2 patients with severe urinary tract infection with poor response to initial medical management. Imaging detection of gas in the renal or perirenal space must encourage aggressive multidisciplinary management including antibiotics and percutaneous drainage or nephrectomy according to the classification and risk factors mentioned, in order to diminish the high morbidity and mortality associated with this disease.

\section{Acknowledgements}

None.

\section{Conflict of interest}

The author declares no conflict of interest. 


\section{References}

1. Nana GR, Brodie A, Akhter W, et al. Nephroureterectomy for emphysematous pyelonephritis: An aggressive approach is sometimes necessary. A case report and literature review. Int J Surg Case. 2015;10:179182.

2. Che-Hung Liu, En-Jui Liu, Yu-Jang Su. Air-fluid level on plain abdominal film: A hint for emphysematous pyelonephritis. Journal of Acute Medicine. 2013;3(1):29-30.

3. Aboumarzouk OM, Hughes O, Narahari K, et al. Emphysematous pyelonephritis: Time for a management plan with an evidence-based approach. Arab Journal of Urology. 2014;12(2):106-115.

4. Uruc F, Yuksel OH, Sahin A, et al. Emphysematous pyelonephritis: Our experience in managing these cases. CUAJ. 2015;9(7):480-484.

5. Garg V, Bose A, Jindal J, et al. Comparison of clinical presentation and risk factors in diabetic and non-diabetic females with urinary tract infection assessed as per the european association of urology classification. $J$ Clin Diagn Res. 2015;9(6):PC12-PC14.
6. Falagas ME, Alexiou VG, Giannopoulou KP, et al. Risk factors for mortality in patients with emphysematous pyelonephritis: a meta-analysis. $J$ Urol. 2007;178(3pt 1):880-885.

7. Eid YM, Salam MM. Diabetic ketoacidosis presenting with emphysematous pyelonephritis. J Diabetes Complications. 2010;24(3):214-216.

8. Turunc T, Kuzgunbay B. The management of emphysematous pyelonephritis and importance of minimally invasive treatment. Urology. 2014;84(4):988.

9. Sang Hyun Park, Ki Hoon Kim. Treatment of a case of emphysematous pyelonephritis that presented with acute abdomen and pneumoperitoneum:a case report. BMC Nephrology. 2015;16:124.

10. Zhang Y, Zang GQ, Tang ZH, et al. Emphysematous Pyelonephritis. Rev Inst Med Trop Sao Paulo. 2015;57(4):368. 